\section{aD VALOREM \\ Journal of Young Researchers}

\section{ACCEso $\mathcal{O}$ abierto}

Para citaciones: Saldarriaga, C., Paba, C. (2020). Caracterización del Comportamiento de la Política Fiscal en Los Ciclos de la Economía Colombiana Entre 2005 y 2020. Revista de jóvenes investigadores Ad Valorem, 3(1), 20-32

Editor: Bernardo Romero Torres. Universidad de Cartagena-Colombia.

Tipología IBN Publindex: Artículo de investigación científica y tecnológica
Copyright: ( 2020. Saldarriaga, C., Paba, C. Este es un artículo de acceso abierto, distribuido bajo los términos de la licencia Creative Commons AtribuciónNoComercial-SinDerivados 4.0 la cual permite el uso sin restricciones, distribución y reproducción en cualquier medio, siempre y cuando que el original, el autor y la fuente sean acreditados.

\title{
Caracterización del comportamiento de la política fiscal en los ciclos de la economía colombiana entre 2005 y 2020
}

\author{
Cristina Saldarriaga Ochoa ${ }^{1}$, Carlos Paba Rivera ${ }^{2}$ \\ Universidad del Atlántico - Colombia
}

\begin{abstract}
RESUMEN
El presente trabajo examina cómo ha respondido la política fiscal en los distintos estados de la economía, específicamente en expansión y recesión entre 2005 y 2020. Para esto se utiliza la desviación del PIB real trimestral con respecto a su tendencia para calcular los ciclos económicos y la variación del déficit en porcentaje del PIB como indicador para caracterizar la actividad fiscal. Sin embargo, considerando que el Estado ejecuta una serie de políticas públicas para contrarrestar los ciclos económicos, que son oscilaciones en la actividad económica, con el fin de promover la estabilidad macroeconómica, que deriva en mantener un crecimiento económico estable y perdurable en el tiempo, los resultados muestran lo variante que ha sido la política fiscal en Colombia. No obstante, aunque anteriormente la política era volátil y estaba fuertemente influenciada por los ciclos de la economía, en los años más recientes, la política se ha mantenido en relativa estabilidad, no importando que el ciclo de la economía se encuentre en recesión.
\end{abstract}

Palabras clave: Ciclo Económico; Déficit Fiscal; Política Fiscal.

\section{Characterization of tax policy behavior in the cycles of the colombian economy between 2005 and 2020}

\begin{abstract}
This paper examines how fiscal policy has responded in different states of the economy, specifically expanding and recession between 2005 and 2020. This uses quarterly real GDP deviation from its trend to calculate economic cycles and deficit variation as a percentage of GDP as an indicator for characterizing fiscal activity. However, considering that the State implements a series of public policies to counter economic cycles, which are fluctuations in economic activity, in order to promote macroeconomic stability, which leads to maintaining stable and lasting economic growth over time, the results show how variant fiscal policy has been in Colombia. However, although politics was previously volatile and heavily influenced by economic cycles, in more recent years, politics has remained relatively stable, no matter that the cycle of the economy is in recession.
\end{abstract}

Key words: Economic Cycle; Fiscal Deficit; Fiscal Policy.

\footnotetext{
${ }^{1}$ Licenciada en Matemáticas y estudiante de Economía de la Universidad del Atlántico. Miembro del Semillero de Mercado Laboral (ORMET- Atlántico).Correo Electrónico: saldarriaga@mail.uniatlantico.edu.co

2 Estudiante de Economía de la Universidad del Atlántico. Miembro del Semillero de Mercado Laboral (ORMET Atlántico).Correo Electrónico: cpaba@est.uniatlantico.edu.co
} 


\section{INTRODUCCIÓN}

En las últimas décadas, el debate sobre el efecto de la política fiscal ha tomado gran relevancia para el área de política económica, esto debido a las recientes crisis internacionales y algunas externalidades que varían el manejo de esta de cualquier país. Una muestra de esto son los Gobiernos de varios países —en especial, EE. UU y Europa - que han implementado políticas fiscales (aumento de gasto, reducción de impuestos, etc.) como instrumentos de gestión pública para estimular la demanda agregada, disminuir el efecto de la recesión, generar empleo e impactar favorablemente el bienestar social de toda la población, en términos de mejoramiento progresivo de la calidad de vida de la sociedad (Sánchez y Galindo, 2013).

Fernández et al., (2006) describen la política fiscal como:

"el conjunto de variaciones en los programas de gastos y de ingresos del gobierno, realizados con el fin de colaborar al logro de los objetivos de la política macroeconómica y ejercer un poderoso impacto sobre la demanda agregada, y, por consiguiente, dado un nivel de precios, sobre la producción y el empleo".

Por su parte, González y Maza (1992)plantean que:

"el propósito de la política fiscal es darle mayor estabilidad al sistema económico, mientras consigue el objetivo de ocupación plena, manteniendo o buscando un punto de equilibrio entre gasto fiscal y el ingreso fiscal, en aras de mantener el balance macroeconómico sin grandes inclinaciones en el empleo ni en la actividad económica, y previniendo severos ataques de inflación. A la vez asegura una adecuada tasa de crecimiento económico mediante una apropiada distribución de la renta entre los diversos grupos de la economía, y una eficiente asignación de recursos en toda la economía".

Teniendo claro lo anterior, el Banco de la República de Colombia menciona que uno de los objetivos de la política fiscal, es moderar los ciclos económicos, que son oscilaciones periódicas de la actividad económica en un periodo determinado, haciendo que el crecimiento económico no sea un proceso lineal de incremento en la cantidad de bienes y servicios producidos, sino que se componga de periodos de expansión o auge, seguido por épocas de recesión, depresión o crisis y recuperación (Padilla, 1986), las cuales son las cuatro fases de un ciclo económico, que no se presentan de la misma forma en diferentes periodos, pues su comportamiento, duración o intensidad pueden cambiar, aunque todos se caracterizan por tener fases descendentes y ascendentes. 
Los ciclos económicos se determinan, principalmente, por el PIB o el PNB, pero las demás variables macroeconómicas también presentan variaciones acordes con cada fase del ciclo, como la inflación y el desempleo. Necesariamente se debe mencionar que, los economistas keynesianos dudan de la capacidad de la economía para alcanzar rápidamente el equilibrio por sí sola, se muestran mucho más inclinados que los clásicos a recomendar que el gobierno actúe para elevar la producción y el empleo durante las recesiones y para moderar el crecimiento económico durante las expansiones.

Por esta razón la política fiscal siempre puede estar orientada en dos maneras de acuerdo: la primera es a actuar de forma contraria u opuesta a la dirección del ciclo económico, es decir contracíclica, y la segunda manera es actuar conforme al ciclo, que se denomina procíclica. Se debe aclarar que la política fiscal de cada periodo depende de la visión que tenga el gobierno de turno; sin embargo, no son estrictamente excluyente porque en algunos casos estos tienen políticas fiscales procíclicas y contracíclica. (Zamudio, 2016).

Por esto, el objetivo de este documento, es el de identificar el comportamiento de la política fiscal en los ciclos económicos de la economía colombiana, con el fin, de obtener un claro panorama de los movimientos dentro de los ciclos de la economía. Por eso, el documento se divide en: aspectos teóricos, dando un breve repaso por la teoría economía, seguidamente, antecedentes y metodología sencilla, utilizando las desviaciones de los ciclos, para determinar el comportamiento de la serie y la variación del déficit con respecto al PIB. Por último, estas los resultados y las conculcaciones.

\section{ASPECTOS TEÓRICOS: LA POLÍTICA FISCAL Y SUS EFECTOS EN LA ECONOMÍA}

Los enfoques teóricos dominantes en la literatura que se han centrado en analizar en qué medida y en que intensidad el Estado debe intervenir en los procesos económicos, así como, los efectos y el impacto que conllevan las decisiones de política fiscal en la actividad real son:

\subsection{Teoría Keynesiana}

Los macroeconomistas clásicos suponen que los precios y los salarios se ajustan rápidamente para igualar la cantidad ofrecida y la demandada en cada mercado, por lo que sostienen que una economía de mercado se "autocorrige" en gran medida, es decir, muestra una clara tendencia a volver por sí sola al equilibrio general, cuando sufre una perturbación económica o hay un cambio de política. Los keynesianos normalmente están de acuerdo en que los precios y los salarios acaban variando lo necesario para equilibrar los mercados; sin embargo, creen que a corto plazo es probable que el ajuste de los precios y de los salarios sea incompleto. Es decir, a corto plazo, las cantidades ofrecidas y las demandadas no 
tienen por qué ser iguales y la economía puede no encontrarse en equilibrio general.

Aunque esta diferencia de puntos de vista parezca puramente teórica, tiene una consecuencia práctica: los keynesianos dudan de la capacidad de la economía para alcanzar rápidamente el equilibrio por sí sola, se muestran mucho más inclinados que los clásicos a recomendar que, el Gobierno actúe elevando la producción y el empleo durante las recesiones, para moderar el crecimiento económico durante las expansiones. (Abel et al., 2004) Por esto, la teoría keynesiana sostiene que el Estado debe mantener un papel activo en la actividad económica, siendo este el principal responsable del desempeño macroeconómico de un país.

Como resultado, el Estado tiene un papel de intervención fuerte, entra como un actor que mitiga los problemas económicos y corrige lo que los economistas denominan "fallas del mercado", los cuales son insuficiencias del sistema económico, que no permiten generan un desarrollo económico óptimo. Por lo cual, los gobiernos a través de la política económica orientan las economías a corregir esos fallos. La intervención se da principalmente por medio del gasto público, la modificación de tasas de interés y el endeudamiento (Stiglitz, 2003).

\subsection{Teoría Neoclásica}

La teoría neoclásica busca reconciliar la teoría clásica y la teoría keynesiana, tomando de cada uno postulados con el fin de reformarlos para plantear un nuevo enfoque, pero bajo el principio de equivalencia Ricardiana, que, según los defensores de la hipótesis, la deuda pública e impuestos son equivalentes, lo que manifiesta que la manera de financiar el gasto público es irrelevante para la economía. Es decir, el efecto sobre la demanda agregada de una variación del gasto es el mismo, tanto si se financia con deuda, como con impuestos.

Dicha teoría fue desarrollada por Ricardo (1820), pero solo expuso las razones de dicha tesis y la desarrolló de forma teórica, lo que le llevó a cuestionar y a rechazar su propia teoría. Fue Barro (1974; 1989) quien relanzó esta teoría, modelizó la equivalencia y la completó con consideraciones como las herencias; de donde resulta que, las decisiones de política fiscal, el gasto, la financiación del gasto y el déficit del sector público, son modificaciones de las composiciones del consumo y el ahorro total de la economía, aunque no generan cambios significativos debido a las expectativas racionales de los agentes económicos. (Fernández, 2019)

Dado que el enfoque neoclásico asume flexibilidad de precios y pleno empleo, los cambios en la producción son el resultado de shocks de oferta. En otras palabras, este enfoque excluye la posibilidad de que la política fiscal pueda tener algún efecto crecimiento a través de la demanda agregada, la política fiscal solo 
afectara la tasa de crecimiento económico a largo plazo, sea por medio de algunos impuestos o de algunos tipos de gasto público que puedan influir en las decisiones de las empresas privadas de invertir en capital humano, conocimiento o investigación y desarrollo, debido a que estas son el motor del crecimiento en una economía. (Narváez, 2017)

\subsection{Teoría Neokeynesiana}

Los neokeynesianos procuran remediar el efecto negativo de las compras del Gobierno en el consumo privado, encontrado en el enfoque neoclásico. Para esto, algunos autores explican la determinación del nivel de precios y el papel de la política fiscal, en relación con el dinero y los precios que se obtiene a partir de la oferta y demanda agregada, derivado de un modelo de equilibrio general dinámico con rigidez nominal de precios y salarios basado en la maximización de los agentes económicos. De acuerdo a Carrillo (2017):

"Ellos encuentran que un incremento del gasto tiene un efecto positivo en el PIB, mientras que los impuestos tienen el efecto contrario; la expansión de las compras gubernamentales tiene un efecto positivo en el consumo de hogares; el aumento conjunto del gasto y los tributos provocan que la inversión privada caiga; incluir la anticipación de los agentes ante la política fiscal no altera las conclusiones [...]".

Hay que mencionar además la escuela austriaca, que sostiene una posición contraria. Es decir, el Estado debe abstenerse de ejercer una intervención directa en la economía, ya que, se regula sola, por medio de la ley de oferta y demanda. Además, el papel del gobierno en los procesos económicos distorsiona la actividad económica y desincentiva la iniciativa privada, causando desequilibrios en la economía. (Heilbroner, 1985, pág 81.)

Sin embargo, a pesar de las diferencias en las posturas sobre la intervención del Estado en la economía, es importante aclarar que las acciones de los Estados están sujetas a las fluctuaciones de los mercados, donde los gobiernos utilizan mecanismos de respuesta, de carácter fiscal o monetario, para intervenir según el ciclo económico. Estos mecanismos, mejor conocidos como los "estabilizadores automáticos", tiene el propósito de moderar dichos ciclos.

Es importante aclarar que para esta investigación solo se analizará el comportamiento de la política fiscal, debido a que este instrumento es la manifestación de la intervención del Estado en la economía, donde, no es un simple regulador de la actividad económica, sino que toma un papel activo en los procesos económicos, a través de gasto e impuestos, que condicionan todo el sistema económico en los países. 


\section{ANTECEDENTES}

Para evidenciar los efectos de la política fiscal en la economía, se tiene en el ámbito internacional, la investigación realizada por García y León (2018), donde analizan la incidencia de la política fiscal en el comportamiento de los ciclos económicos de Ecuador para el periodo 2000 a 2016, a través de un modelo econométrico VAR, tomando las variables, ratio de deuda y la desviación del PIB real con respecto a su tendencia, se concluye que la relación entre déficit fiscal y crecimiento económico es inversa en promedio; es decir, incrementos del déficit público provocan contracciones económicas en los próximos periodos, tanto en situaciones de expansión o contracción económica. Además, incrementos del déficit público no generan procesos inflacionarios dentro del régimen monetario dolarizado sino por emisiones inorgánicas, debido principalmente a la pérdida de capacidad de emisión monetaria.

En el ámbito nacional y en consonancia con la primera investigación, Zamudio (2016), realiza una aproximación hacia los procesos de estabilización macroeconómica que tiene el Estado Colombiano, como manera de intervenir en la economía a través de la política fiscal. Especialmente, la manera como el gobierno de Juan Manuel Santos (periodo 2010-2014) actuó por medio de la Política Fiscal en cuestión de gasto público y deuda pública, para afrontar los ciclos de la economía y qué repercusiones tuvo en el desempeño económico del país. Para esto, utilizo la variación porcentual del PIB para observar las fluctuaciones que ha tenido la economía Colombia y el déficit fiscal para mirar el comportamiento de la política fiscal. Como resultado, ostentó que la política fiscal se manejó de las dos maneras (contracíclica y procíclica), y que, a pesar de las crisis económicas internacionales, Colombia mantuvo una cierta estabilidad macroeconómica en el cuatrienio.

En el ámbito local se hace mención a Baca (2010),quien identifico en qué momento la política fiscal en Colombia fue anticíclica o procíclica en los años 1950-2004 y 1962-2004, considerando para el primer período el Déficit del Sector Público No Financiero (SPNF) y para el segundo periodo, el Déficit del Gobierno Nacional Central (GNC). Esta investigación, se logra identificar que los periodos anticíclicos, estuvieron acompañados con bajas tasas de desempleo; y, los periodos procíclicos por altas tasas de desempleo. Las estimaciones econométricas, mediante modelos tipo VAR, mostraron que el déficit fiscal, tanto del SPNF como del GNC, generan efectos reales positivos en el corto plazo sin desestabilización de precios, y se concluye que la política fiscal debe tener discrecionalidad para reactivar la economía cuando se desacelere y el sector externo no tenga restricciones como la devaluación de la moneda local originada por desbalances externos negativos. Igualmente, la soberanía monetaria representa la clave para un programa macroeconómico que garantice el pleno empleo y la estabilidad de precios. 


\section{METODOLOGÍA}

Para determinar el comportamiento de los ciclos económicos y sus fluctuaciones, se utiliza la metodología descrita por Padilla (1986) la cual consiste en medir la desviación del PIB real trimestral -para evitar fluctuaciones estacionales- con respecto a la tendencia o promedio registrado en el periodo de tiempo que se está analizando. Se utiliza la información del PIB real calculado por el Departamento Administrativo Nacional de Estadística (DANE) en forma trimestral con periodo base de 2015 ; mientras que los datos de la Política Fiscal del gobierno se toman de las cifras oficiales que recoge el Banco de la República a nivel mensual, estos datos son sumados a niveles de trimestres para que concuerden con la base del PIB real.

Seguidamente, se calculó la línea de tendencia trimestral del PIB real en el periodo trimestral de los años 2005 al 2020, para luego obtener los valores de la proyección del PIB que pertenecen a la línea de tendencia y computar la desviación de los datos del PIB real con los de la proyección. Este resultado es la fluctuación cíclica del PIB la cual determina el ciclo económico de un país. Para calcular el comportamiento de la política fiscal adoptada por el gobierno en función de la fase del ciclo económico, se emplea las diferencias trimestrales del déficit en porcentaje de PIB debido a que muestra la tasa de variación (incremento o disminución) del déficit en porcentaje de PIB.

$$
C=\left[\frac{\text { Déficit }}{P I B}\right]_{t}-\left[\frac{\text { Déficit }}{P I B}\right]_{t-1}
$$

Ahora bien, al momento de que los responsables de la ejecución de la política fiscal deban tomar decisiones sobre los ciclos económicos, tendrán que decidir entre una política fiscal con comportamiento procíclico o anticíclico (Burns y Mitchell, 1946). De acuerdo a Burns y Mitchell (1946):

"Si el ciclo económico es recesivo y el Gobierno decide reducir el gasto público o aumentar los impuestos, entonces, la política fiscal será procíclica, es decir, a medida que la producción, los ingresos y el empleo disminuyen, el aumento de los impuestos, por ejemplo, reduce el ingreso disponible, lo que refuerza la caída de estas variables. En otras palabras, una política fiscal procíclica acentúa la tendencia, en este caso recesiva, del ciclo económico."

Por otro lado, si el ciclo económico es recesivo y el gobierno decide aumentar el gasto público y reducirlos impuestos, en este caso, la política fiscal será anticíclica. En conclusión, si la tendencia de la variación de la política fiscal es la misma que la del ciclo económico, esta se considerará como procíclica, de lo contrario será de tipo anticíclica. (Baca, 2010) 
Para identificar los periodos en que la política fiscal fue procíclica o anticíclica se debe tener en cuenta la siguiente regla de interpretación: cuando el ciclo económico y $\triangle$ Déficit/PIB se mueven en la misma dirección, la política fiscal es procíclica. En caso de que se mueva en sentido contrario, el comportamiento de la política fiscal es anticíclico.

Por otro lado, es importante tener en cuenta que la política fiscal, puede ser anticíclica y al mismo tiempo expansiva como contractiva. Será expansiva cuando el ciclo económico se reduzca y el $\triangle$ Déficit/PIB aumente. Será contractiva cuando el ciclo económico aumente y el $\Delta$ Déficit/PIB disminuya. De igual forma, la política fiscal procíclica puede ser expansiva o contractiva. Será expansiva cuando el ciclo económico se incremente y el $\triangle$ Déficit/PIB igualmente lo haga. Será contractiva cuando el ciclo económico se reduzca y el $\triangle$ Déficit/PIB de igual forma lo haga. En resumen, la anticíclica busca estabilizarlas fluctuaciones del ciclo económico. La procíclica profundiza dichas fluctuaciones (Baca, 2010).

\section{RESULTADOS}

Debido a el objetivo del trabajo, no se busca identificar eventos de causalidad, entre los ciclos económicos y la evolución de la política fiscal del país, es entendible que, lo eventos históricos y espaciales, son determinantes del comportamiento de ambos indicadores. Pero, en concordancia con la razón de ser de este estudio, solo se limita a la caracterización de la política fiscal en los ciclos económicos del país.

Al aplicar la metodología propuesta por Baca, (2010), la gráfica 1 muestra las fluctuaciones del ciclo económico junto al comportamiento de la política fiscal. En ella, se puede identificar, qué, durante el periodo analizado, en Colombia se han presentado dos ciclos económicos. Como se mencionó en la literatura, para determinar un ciclo económico completo se toma como punto de inicio la etapa de auge y como fin, la fase de recuperación (Abel et al., 2004).

Grafica 1. Comportamiento trimestral del $\triangle$ Déficit/PIB y Ciclo Económico

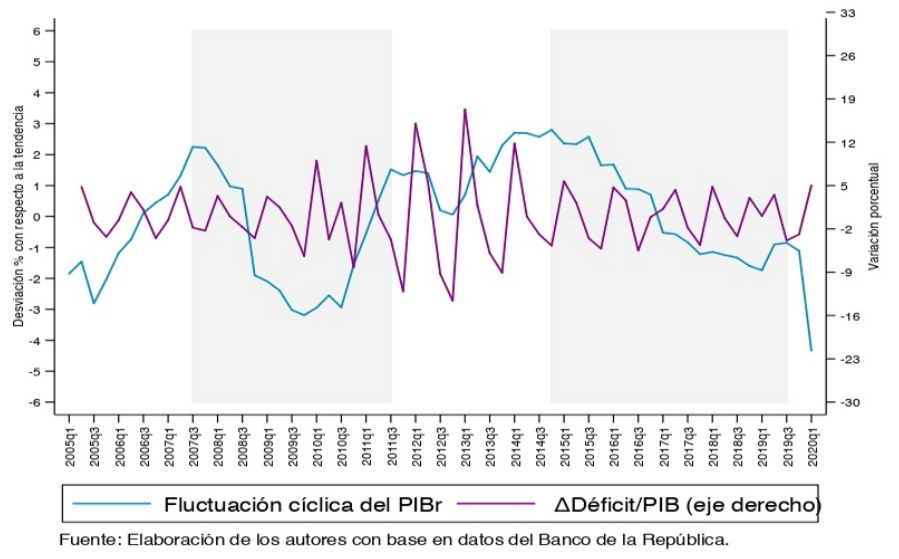


Los tres primeros años, sin incluir los dos últimos trimestres de 2007, el país muestra una recuperación; por esto, el primer ciclo económico inicia en el tercer trimestre de 2007 y concluye en el segundo trimestre del 2011; mientras que el segundo ciclo abarca el tercer trimestre de 2011 hasta el tercer trimestre del 2014, periodo en el cual se evidencia una breve recesión en el año 2012, seguida de un repunte vertiginoso de recuperación y auge para la economía colombiana, por eso no se considerara como una fase del ciclo. En el cuarto trimestre de 2014 inicio un nuevo ciclo económico que alcanzo la fase de crisis durante el 2018 y una tentativa recuperación para el 2019. No obstante, para el último trimestre de 2019 y el primero del 2020 la economía entro en una depresión.

Ahora bien, la tabla 1, expone la dinámica de respuesta de la política fiscal y el tipo de política para las fluctuaciones del ciclo de la economía colombiana. Se tiene que, para los tres primeros años en donde se ve un periodo de depresión hasta auge en las fases del ciclo, la política fiscal fue variante siendo procíclica y expansiva en los primeros trimestres de los años, tanto en recuperación como en auge. No obstante, en la segunda mitad de los años, la política se comportó de manera anticíclica y contractiva.

En el año 2008, Colombia estaba en una recesión que se agravo hasta el 2009, en ese periodo la política fiscal fue procíclica y contractiva, para luego en el periodo de recuperación del 2010 pasar a contracíclica en los tres primeros trimestres y alternar entre contractiva y expansiva en los trimestres respectivamente. Para los años siguientes la política fiscal varia de un periodo a otro, mientras que la economía entra en una breve recesión, recuperándose rápidamente, en todos estos casos, la política se manutuvo procíclica, desde finales del 2011 hasta el tercer trimestre del 2013. Desde allí, al parecer la política fiscal entra en un periodo de relativa estabilidad. Siendo procíclica en los 3 primeros trimestres y anticíclica en el último trimestre del año, esto es desde el 2014 al 2016, reactivándose en 2018 hasta el 2019. El año 2017, la política fiscal, fue contracíclica en el primer trimestre y procíclica en los demás periodos.

Desde el auge que se tuvo en el 2014, la economía colombiana experimento una recesión que finaliza con una depresión en el año 2018, se tiene un indicio de recuperación en el 2019 que luego es fuertemente truncado por la coyuntura internacional que vivía en ese momento. En cuanto al tipo de política implementado por el Gobierno de turno, esta fue coyuntural y cambiante para cada trimestre, pero en el primer trimestre del 2020 el gobierno empleó una política fiscal expansiva. 
Tabla 1. Fase del Ciclo y Movimientos de la Política Fiscal

\begin{tabular}{|c|c|c|c|}
\hline Ã̃̃ & $\begin{array}{l}\text { FASE DEL } \\
\text { CICLO }\end{array}$ & POLITICA FISCAL & TIPO DE P. FISCAL \\
\hline 2005 & depresión & procíclica & contractiva \\
\hline 2006 & recuperación & $\begin{array}{l}\text { I, II y procíclica, III y IV } \\
\text { anticíclica }\end{array}$ & $\begin{array}{l}\text { I y II expansiva, III y } \\
\text { IV contractiva }\end{array}$ \\
\hline 2007 & auge & $\begin{array}{l}\text { I procíclica, II, III y IV } \\
\text { anticíclica }\end{array}$ & $\begin{array}{l}\text { I expansiva, II, III y IV } \\
\text { contractiva }\end{array}$ \\
\hline \multicolumn{4}{|c|}{$\begin{array}{c}\text { para pasar del último trimestre } 2007 \text { al primer trimestre } 2008 \text {, la política fiscal fue } \\
\text { contracíclica }\end{array}$} \\
\hline 2008 & recesión & procíclica & contractiva \\
\hline \multicolumn{4}{|c|}{$\begin{array}{l}\text { para pasar del último trimestre } 2008 \text { al primer trimestre } 2009 \text {, la política fiscal fue } \\
\text { contracíclica }\end{array}$} \\
\hline 2009 & depresión & procíclica & contractiva \\
\hline 2010 & recuperación & $\begin{array}{l}\text { I y III contracíclica, II, IV } \\
\text { procíclica }\end{array}$ & $\begin{array}{l}\text { I contractiva, II } \\
\text { expansiva, III } \\
\text { contractiva y IV } \\
\text { expansiva }\end{array}$ \\
\hline 2011 & auge & $\begin{array}{l}\text { I, II y III contracíclica, IV } \\
\text { procíclica }\end{array}$ & contractiva \\
\hline 2012 & $\begin{array}{l}\text { recesión y } \\
\text { depresión }\end{array}$ & Procíclica & $\begin{array}{l}\text { I, II y III contractiva, } \\
\text { IV expansiva }\end{array}$ \\
\hline 2013 & recuperación & procíclica & $\begin{array}{l}\text { I y II contractiva, III y } \\
\text { IV expansiva }\end{array}$ \\
\hline 2014 & auge & $\begin{array}{l}\text { I, II y III procíclica, IV } \\
\text { anticíclica }\end{array}$ & $\begin{array}{l}\text { I, II y III contractiva, } \\
\text { IV expansiva }\end{array}$ \\
\hline 2015 & recesión & $\begin{array}{l}\text { I, II y III procíclica, IV } \\
\text { anticíclica }\end{array}$ & $\begin{array}{l}\text { I, II y III contractiva, } \\
\text { IV expansiva }\end{array}$ \\
\hline 2016 & recesión & $\begin{array}{l}\text { I, y II procíclica, III y IV } \\
\text { anticíclica }\end{array}$ & $\begin{array}{l}\text { I y II contractiva, III y } \\
\text { IV expansiva }\end{array}$ \\
\hline 2017 & recesión & $\begin{array}{l}\text { I contracíclica, II, III y IV } \\
\text { procíclica }\end{array}$ & $\begin{array}{l}\text { I expansiva, II, III y IV } \\
\text { contractiva }\end{array}$ \\
\hline \multicolumn{4}{|c|}{$\begin{array}{c}\text { para pasar del último trimestre } 2017 \text { al primer trimestre } 2018 \text {, la política fiscal fue } \\
\text { contracíclica }\end{array}$} \\
\hline 2018 & depresión & $\begin{array}{l}\text { I procíclica, II, III y IV } \\
\text { anticíclica }\end{array}$ & $\begin{array}{l}\text { I contractiva, II, III y } \\
\text { IV expansiva }\end{array}$ \\
\hline 2019 & recuperación & $\begin{array}{c}\text { I procíclica, II, III y IV } \\
\text { anticíclica }\end{array}$ & $\begin{array}{l}\text { I contractiva, II, III y } \\
\text { IV expansiva }\end{array}$ \\
\hline 2020 & recesión & I anticíclica & I expansiva \\
\hline
\end{tabular}




\section{CONCLUSIONES}

Es importante recalcar que Colombia desde la misma constitución de 1991 manifiesta su posibilidad de intervención en la economía, como un Estado que toma la dirección general del comportamiento de la misma, y el articulo 334 deja establecida la necesidad de mantener una sostenibilidad fiscal para proteger los intereses económicos del país.

Por esto, el objetivo del trabajo es determinar el comportamiento de la Política Fiscal en los ciclos económicos de la economía colombiana. Para esto se utilizó la información del PIB real, calculada por el DANE, información que se brinda a la opinión pública de forma trimestral con año base de 2015 para calcular la línea de tendencia trimestral del PIB de acuerdo a la metodología descrita por (Padilla, 1986). En cuanto al cálculo del comportamiento de la Política Fiscal, se emplean las diferencias trimestrales en puntos porcentuales del déficit como porcentaje del PIB.

De acuerdo a esto, al comparar ambos indicadores se determinó que la política fiscal ha sido tanto procíclica como contracíclica, pero en general, durante el periodo analizado, la política fiscal dominante fue la procíclica, lo que quiere decir que va en el mismo sentido del ciclo económico del país, si bien la situación económica de un país se encuentra en un auge, esta política sigue incentivando esta situación. Sin embargo, si el país se encuentra en un periodo de recesión económica, la política fiscal disminuye.

Con respecto al tipo de política fiscal, la más aplicada es la expansiva para estimular la economía y aumentar la demanda agregada, mediante el aumento del gasto público y la bajada de impuestos. Evidentemente, con la aplicación de estas medidas se generará déficit público. El análisis se concreta, en manera de enfocar la política fiscal del gobierno colombiano ante los movimientos del ciclo económico; esto sirve como insumo fundamental, para identificar la respuesta ante posibles escenarios a futuro, es decir, ante las posibles externalidades o eventos internos que cambien el comportamiento del ciclo y afecten la calidad de vida los ciudadanos.

\section{REFERENCIAS}

Abel, A. B., Bernanke, B. S., Rabasco, E., y Toharia, L. (2004). Macroeconomía (Cuarta). Pearson España. https://www.researchgate.net/profile/Andrew_Abel/publication/48212535_Macroeconomia /links/0c9605331d0cc7b314000000.pdf

Baca Mejía, W. R. (2010). La política fiscal en Colombia:¿ procíclica o anticíclica? [Universidad del Norte]. http://manglar.uninorte.edu.co/handle/10584/2111\#page=1 
Barro, R. J. (1974). Are government bonds net wealth? Journal of Political Economy, 82(6), 10951117. https://www.journals.uchicago.edu/doi/abs/10.1086/260266?journalCode=jpe

Barro, R. J. (1989). The Ricardian approach to budget deficits. Journal of Economic Perspectives, 3(2), 37-54. https://doi.org/10.1257/jep.3.2.37

Burns, A. F., y Mitchell, W. C. (1946). Measuring business cycles. NBER Book Series Studies in Business Cycles. https://www.nber.org/books/burn46-1?mod=article_inline

Carrillo Maldonado, P. A. (2017). El efecto de la política fiscal en expansión y recesión para Ecuador: un modelo MSVAR (The Effect of Fiscal Policy on Expansions and Recessions in Ecuador: A MSVAR Model). Cuadernos de Economía, 36(71). https://ssrn.com/abstract=2926737

Fernández Carmona, A. (2019). La hipótesis de la equivalencia ricardiana. Un análisis empírico: el caso de los países nórdicos [Universidad del Pais Vasco.]. http://hdl.handle.net/10810/31270

Fernández Díaz, A., Parejo Gámir, J. A., y Rodríguez Sáiz, L. (2006). Politica Economica. (J. I. Fernández (ed.); Cuarta). McGraw-Hill. https://es.scribd.com/doc/261839343/PoliticaEconomica-Fernandez-Diaz-4ED

García Ortiz, J. C., y León León, J. J. (2018). Incidencia de la política fiscal en el comportamiento de los ciclos económicos nacionales para el periodo 2000-2016. Quito: UCE. http://www.dspace.uce.edu.ec/handle/25000/15939

González, A., y MAZA, D. (1992). Tratado moderno de economía. In Panao (Ed.), Caracas: Editorial Panapo. https://www.academia.edu/31141497/Tratado_Moderno_de_economia_Maza_Zavala_pdf

Heilbroner, R. L. (1985). Vida y doctrina de los grandes economistas. Orbis. https://sociologiadeldesarrolloi.files.wordpress.com/2014/11/184308128-heilbroner-robertvida-y-doctrina-de-los-grandes-economistas-cap-3-y-4.pdf

Narváez, R. C. (2017). Crecimiento económico y política fiscal: una revisión crítica de la literatura. Ensayos de Economía, 27(51), 79-107. https://doi.org/https://doi.org/10.15446/ede.v27n51.69104

Padilla Aragón, E. (1986). Ciclos económicos y política de estabilización. In Siglo Veintiuno Editores (Ed.), Economía y demografía (Cuarta). Universidad de Michigan.

Ricardo, D. (1820). Essay on the Funding System. The Works of David Ricardo. McCulloch ed, 1888. John Murray. https://oll.libertyfund.org/titles/ricardo-the-works-of-david-ricardomcculloch-ed-1846-1888

Sánchez, W., y Galindo, H. (2013). Efectos simétricos y asimétricos de la política fiscal en el Perú (Informe final PB17-2011). Universidad Nacional de Ingeniería. https://www.cies.org.pe/es/investigaciones/politica-macroeconomica-ycrecimiento/efectos-simetricos-y-asimetricos-de-la 
Stiglitz, J. E. (2003). La economía del sector público (Vol. 24). Antoni Bosch Editor. https://books.google.es/books?hl=es\&|r=\&id=uJmFnvYxDBEC\&oi=fnd\&pg=PA1\&dq=La+ economía+del+sector+público+\&ots=LDv7JOuuLz\&sig=sAYhDiAjpt8JNZOTXXK1RfuJ5s\#v=onepage\&q=La economía del sector público\&f=false

Zamudio González, L. (2016). La política fiscal en los ciclos de la economía colombiana [Universidad del Rosario]. http://repository.urosario.edu.co/handle/10336/12894 